
S. J. Lindenbaum, H. Okuno, ${ }^{*}$ Y. Teramoto, ${ }^{\dagger}$ C. D., Wheeler, and A. H. Walenta Brookhaven National Laboratory, Upton, New York

$$
\begin{gathered}
\downarrow \text { H. B. Jensen }{ }^{\ddagger} \text { and B. Sadoulet } \\
\text { CERN, Geneva, Switzerland }
\end{gathered}
$$

M. Atac

Fermi National Accelerator'Laboratory, Batavia, Illinois

$$
\text { R: Stump }
$$

-University of Kansas, Lawrence, Kansas

\title{
A. Kanof sky
}

Lehigh University, Bethlehem, Pennsylvania

R. Weinstein

Northeastern University, Boston, Massachusetts

s. White

$\backslash$ Rockefeller University, New York, New York

At the 1977 ISABEIIE Summer Workshop, attempts have been made to predict the behavior of proportional wire chambers at the high particle flux expected for ISABELIE. I It has been found that chambers running at presently widely used high gas gain would have impaired performance concerning life time, efficiency, stability of gas gain and position resolution. In the mean time more information has become available and therefore these properties are revised in the present study.

An improvement can also be expected for much lower gas gain, a possibility which is investigated here in more detail for its consequences on position resolution and time resolution.

*on leave from Institute for Nuclear Study, University of Tokyo, Tanashi, Tokyo, Japan. ton leave from Osaka City University, Osaka, Japan. Fon leave from Niels Bohr Institute, Copenhagen, Denmark.

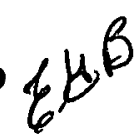

There is no objectlon from the patew point of viow to the pybitcertion disespintion of the documental untors in this lettor. 


\section{DISCLAIMER}

This report was prepared as an account of work sponsored by an agency of the United States Government. Neither the United States Government nor any agency Thereof, nor any of their employees, makes any warranty, express or implied, or assumes any legal liability or responsibility for the accuracy, completeness, or usefulness of any information, apparatus, product, or process disclosed, or represents that its use would not infringe privately owned rights. Reference herein to any specific commercial product, process, or service by trade name, trademark, manufacturer, or otherwise does not necessarily constitute or imply its endorsement, recommendation, or favoring by the United States Government or any agency thereof. The views and opinions of authors expressed herein do not necessarily state or reflect those of the United States Government or any agency thereof. 


\section{DISCLAIMER}

Portions of this document may be illegible in electronic image products. Images are produced from the best available original document. 
The expected high multiplicity of tracks belonging to a single event, the high event rates and the requirement of low gas gain request a revision on the methods for the measurement of the second coordinate.

Particle identification measuring the relativistic rise of energy loss in the chambers has been investigated in more detail than in previous ISABELIE. summer studies using new data and calculations.

\section{RATE EFFECTS IN PROPORTIONAL CHAMBERS AND DRIFT CHAMBERS}

High particle rates at ISABELLE raise fundamental questions on the operation of proportional and drift chambers, as e.g., discussed by A. H. Walenta in the Proceedings of the ISABELLE Workshop 1977. Here we summarize results on the chamber life time based on new measurements and more detailed considerations of space charge effects and gain saturation.

\section{Particle Rates}

Charged particle rates can be estimated according to calculations by F. E. Paige. ${ }^{2}$ A simple approximation to the cross section curve at $\sqrt{\mathrm{s}}=800 \mathrm{GeV}$ gives

$$
\begin{array}{lll}
\frac{d \sigma}{d \Omega}=8 \times 10^{-27} / e^{1.9} & \left(\mathrm{~cm}^{2} \cdot \mathrm{sr}^{-1}\right) & \text { for } 10^{-3} \leq \theta \leq 0.5 \\
\frac{d \sigma}{d \Omega}=13 \times 10^{-27} / 0^{1.2} & \left(\mathrm{~cm}^{2} \mathrm{sr}^{-1}\right) & \text { for } 0.5 \leq \theta \leq \pi / 2 .
\end{array}
$$

For a iluinosity $L$, the particle rate in solid angle $\Delta \Omega$ is given by

$$
R: \equiv \equiv=L \frac{\mathrm{d} O}{\mathrm{~d} \Omega} \cdot \Delta \Omega \quad\left(\text { particle.s } s^{-1}\right)
$$

The partie rate per unit wire length of the chamber depends on the configuration and gec: $:=$ ises of the chamber. Typical examples are shown in Fig. 1, where the scattering engle $\theta$ is divided into three regions. 
Region I $\left(30^{\circ} \leq \partial \leq 150^{\circ}\right)$ is covered by a "bicycle wheel" drift chamber, which is segmented in $N$-sections. The particle rate per unit wire length $(1 \mathrm{~cm})$ is $^{3}$

$$
\left.\mathrm{n}=\mathrm{L} \frac{\mathrm{d} \sigma}{\mathrm{d} \Omega} \cdot \frac{1}{\mathrm{r}} \cdot \frac{2 \pi}{\mathrm{N}} \cdot \sin ^{3} \theta \quad \text { (particles.cm }{ }^{-1} \cdot \mathrm{s}^{-1}\right) \quad
$$

The life time of the chamber and space charge effects are considered to depend on the product of the number of particles and the size of the avalanche. When we assume that the size of the avalanche for the particle track perpendicular to the anode wire is $q_{0}$, the avalanche size for the inclined track is given by $q=q_{0} / \sin \theta$. Then the charge rate $Q$ per unit wire length is

$$
Q=n \cdot q=I \frac{d o}{d z} \cdot \frac{1}{r} \cdot \frac{2 \pi}{N} \sin ^{2} \theta \cdot q_{0} \quad\left(\text { electrons } \cdot \mathrm{cm}^{-1} \cdot \mathrm{s}^{-1}\right) \quad .
$$

$Q$ along the anode wire changes only by a factor of 2 in this region, and is almost constant at small $\theta$ because of Eq. (1). The maximum $Q$ is at $\theta=30^{\circ}$, and for $L=$ $5 \times 10^{32} \mathrm{~cm}^{-2} \cdot \mathrm{s}^{-1}$

$$
Q_{\max }=2.2 \times 10^{7} \frac{q_{0}}{\Gamma N} \quad\left(\text { electrons } \mathrm{cm}^{-1} \cdot \mathrm{s}^{-1}\right) \text {. }
$$

In order to get the optinum value of $Q_{\max }$, we have three adjustable parameters $r, N$ and $q_{0}$. For $r=10 \mathrm{~cm}, N=90$ and $q_{o}=2 \times 10^{6} \mathrm{e}, Q_{\max }=4.8 \times 10^{10} \mathrm{e} \cdot \mathrm{cm}^{-1} \cdot \mathrm{s}^{-1}$, and the rate per wire is about $10^{6}$ particles.s ${ }^{-1}$.

Since the particle rate increases at smaller angles, region II $\left(10^{\circ} \leq \theta \leq 30^{\circ}\right)$ is covered by circuiar, flat drift chambers with relatively narrow drift space. With the drift space d, the particle rate per unit length of the wire is

$$
\mathrm{n}=\mathrm{L} \cdot \frac{\mathrm{d} \sigma}{\mathrm{d} \Omega} \frac{\mathrm{d}}{\mathrm{r}^{2}} \sin ^{2} \theta \cdot \cos \theta \quad\left(\text { particles.cm } \mathrm{cm}^{-1} \cdot \mathrm{s}^{-1}\right)
$$

\footnotetext{
We consider here the expected luminosity in the low $\beta$ configuration.
} 
At $\theta=10^{\circ}$ and $\mathrm{L}=5 \times 10^{32} \mathrm{~cm}^{-2} \cdot \mathrm{s}^{-1}$,

$$
Q_{\max }=3.2 \times 10^{6} \frac{\mathrm{d}}{\mathrm{r}^{2}} \cdot \mathrm{q}_{\mathrm{o}} \quad\left(\text { electrons.cm } \mathrm{cm}^{-1} \mathrm{~s}^{-1}\right. \text { ) }
$$

For $\mathrm{r}=10 \mathrm{~cm}, \mathrm{~d}=0.5 \mathrm{~cm}$ and $\mathrm{q}_{0}=2 \times 10^{6} \mathrm{e}, Q_{\max }=3.2 \times 10^{10} \mathrm{e} \cdot \mathrm{cm}^{-1} \cdot \mathrm{s}^{-1}$.

In region III $\left(\theta \leq 10^{\circ}\right), d \sigma / d \Omega$. is roughly proportional to $1 / \theta^{2}$ and $\sin ^{2} \theta \cos \theta=$ $\theta^{2}$. Therefore, particle rate is simply. expressed as (see also Ref. 4)

$$
\left.\mathrm{n}=3.2 \times 10^{6} \frac{\mathrm{d}}{\mathrm{r}^{2}} \quad \text { (particles } \mathrm{cm}^{-1} \cdot \mathrm{s}^{-1}\right)
$$

For $r=3 \mathrm{~cm}, \mathrm{~d}=0.2 \mathrm{~cm}$ and $\mathrm{q}_{\mathrm{o}}=2 \times 10^{6} \mathrm{e}$, one obtains $\mathrm{n}=7 \times 10^{4}$ particles $\cdot \mathrm{cm}^{-1} \cdot \mathrm{s}^{-1}$ and $Q_{\max }=1.4 \times 10^{11} \mathrm{e}^{\mathrm{cm}} \mathrm{cm}^{-1} \mathrm{~s}^{-1}$. When chambers are placed at $\mathrm{z}=500 \mathrm{~cm}$, the minimum angle covered is $\theta=0.006$.

\section{Life Time}

The life time of the chamber is limited due to the polymerization effect of some quenchers or impurities. During long-exposures to the intense radiation, deposits of polymers are formed on the anode and on the cathode, which decrease gas gain and deteriorate the energy resolution and increases background.

Accumulation of a critical amount of deposits is, in simple approximation, considered to be proportional to the integral amount of free charges created in the avalanche per urit wire length. The definition of a critical value, $Q_{c r i t}$ ' for deterioration of the chamber depends on the operation mode of the chamber. When the chamber is used for $d E / d x$ measurement, gain variations of a $f$ ew $\%$ cause problems. On the other hand, when it is used only for position readout, requirement for energy resolution is more relaxed. 
In the 1977 Workshop, $Q_{\text {crit }}$ was estimated to be $Q_{\text {crit }}=2.5 \times 10^{17} \mathrm{e} \cdot \mathrm{cm}^{-1}$ based on Geiger counter data. To check the validity of this assumption, new measurements have been carried out at BNL. ${ }^{5}$ preliminary results are shown in Fig. 2, where the energy resolution for ${ }^{55} \mathrm{Fe} x$-rays $(5.9 \mathrm{keV})$ is plotted as a function of the accumulated free charge. When we define $Q_{c r i t}$ as the integrated charge where the energy resolution changes as indicated in Fig. 2. Qcrit for various gas mixtures can be compared as shown in Table 1.

Table 1. Q

\begin{tabular}{l|l}
\hline \multicolumn{1}{c|}{ Gas Mixtures } & Q $_{\text {crit }}\left(\mathrm{e}^{\mathrm{cm}}{ }^{-1}\right)$ \\
\hline $\operatorname{Ar}(90 \%)+\mathrm{CH}_{4}(10 \%)$ & $5.3 \times 10^{18}$ \\
\hline $\operatorname{Ar}(70 \%)+\mathrm{Isobutane}(30 \%)$ & $5.4 \times 10^{18}$ \\
\hline $\operatorname{Ar}(67.2 \%)+$ Isobutane $(28.8 \%)+$ Methylal $(4 \%)$ & $3.3 \times 10^{19}$ \\
\hline $\operatorname{Ar}(80 \%)+\mathrm{CO}_{2}(20 \%)$ & $>10^{20}$ \\
\hline
\end{tabular}

The best result is abtained for the $\mathrm{Ar} / \mathrm{CO}_{2}$ mixture. This mixture may be considered if necessary but needs some further investigation concerning its behavior in drift chambers. For Ar/Isobutane, addition of a small amount of Methylal improves the counter life appreciably. By using the value for Ar/ Isobutane/Methylal, the life time of chambers described in the previous section can be estimated. Results are shown in Table 2. 
Table 2. Life time of chambers. $L=5 \times 10^{32} \mathrm{~cm}^{-1} \cdot \mathrm{s}^{-1}, \mathrm{q}_{0}=2 \times 10^{6} \mathrm{e}$ and

$$
Q_{\text {crit }}=3 \times 10^{19} \mathrm{e} \cdot \mathrm{cm}^{-1} \text { are assumed. }
$$

\begin{tabular}{|c|c|c|c|c|c|}
\hline & Type of Chamber & $r_{\min }(\mathrm{cm})$ & $\begin{array}{l}\text { Maximum Particle } \\
\text { Rate } \mathrm{n}_{\max } \\
\left(\mathrm{cm}^{-1} \cdot \mathrm{s}^{-1}\right)\end{array}$ & $\begin{array}{l}Q_{\max }=n \cdot q_{o} \\
\left(e \cdot c m=s^{-1}\right)\end{array}$ & $\begin{array}{l}\text { Life Tine } \\
\text { (years) }\end{array}$ \\
\hline Region I & $\begin{array}{c}\text { Bicycle Wheel } \\
\text { Drift Chamber } \\
\mathrm{N}=90\end{array}$ & 10 & $2.4 \times 10^{4}$ & $4.8 \times 10^{10}$ & 20 \\
\hline Region II & $\begin{array}{l}\text { Circular, Flat } \\
\text { Drift Chamber } \\
\mathrm{d}=0.5 \mathrm{cr} \\
\end{array}$ & 10 & $1.6 \times 10^{4}$ & $3.2 \times 10^{10}$ & 30 \\
\hline Region III & $\begin{array}{l}\text { PWC } \\
\quad d=0.2 \mathrm{~cm}\end{array}$ & 3 & $7.0 \times 10^{4}$ & $1.4 \times 10^{11}$ & 7 \\
\hline
\end{tabular}

These numbers seem to be very encouraging. It should be noted that the assumed value of $Q_{\text {crit }}$ was obtained with a small, cylindrical test tube and with the clean gas system. Further study on the effect of wire cathodes and effect of impurities should be made.

\section{Space Charge Effect}

In $\mathrm{d} E / \mathrm{dx}$ measurements for particle identification, the gain shift of the chamber due to the space charge of positive ions is a serious problem at high rates. With a typical geometry of the drift chamber $(d=1 \mathrm{~cm}), 2 \%$ gain shift was observed at $Q=5 \times 10^{10} \mathrm{e} \cdot \mathrm{cm}^{-1} \cdot \mathrm{s}^{-1}$. Recent results at $\mathrm{CERN}^{6}$ also showed the gain shift of about $2 \%$ at $Q=10^{10} \mathrm{e} \cdot \mathrm{cm}^{-1} \cdot \mathrm{s}^{-1}$ for the drift chamber of $\mathrm{d}=2 \mathrm{~cm}$. These numbers are compatible to $Q_{\max }$ for each configuration of chambers given in Table 2 . In Region II and Region III, smaller drift distances are chosen, so that the space charge effect will be much reduced. Therefore, chamber geometries considered in Table 2 seem to be acceptable. 
When a relatively long drift space is required, a possible solution to reduce the space charge effect is the introduction of grid wires near the anode wire. In Fig. 3, two examples are shown. The effect of this grid would be two-fold; it reduces the number of positive ions reaching the long drift region, and for positive ions escaped to the drift region, the shielding effect of the grid reduces their influence on the field near the ariode. The fraction of positive ions escaping to the drift region depends on two factors; the field shape near the grid, and the azimuthal spread of positive ions around the anode wire. The latter is about $100^{\circ}$ (FINRM) in the proportional region of gas amplification for $\operatorname{Ar}(90 \%)+$ $\mathrm{CH}_{4}(10 \%)$, and changes slightly depending on the amount of quencher. 7 As the avalanche is relatively well localized on one side of the wire, grid spacing should be relatively narrow compared to the grid-anode distance. By choosing a suitable potential, one can get the fraction escaping to be $0.1-0.5$. The TPC-group ${ }^{8}$ measured a value of less than 0.1 for the geometry given in Fig. 3(b). For these grid planes placed at $5 \mathrm{~mm}$ from the anode plane, a rate behavior equivalent to the drift chamber of $\mathrm{d}=1 \mathrm{~cm}$ can be expected.

Another new approach to overcome high rate limitation was proposed recently by G. Charpak et al. ${ }^{9}$ using a preamplification zone, a drift delay, a gate (chamber requires a trigger) and a final amplification zone. The space charge effect in the final amplification zone is reduced if the number of selected events is much smaller compared to the overall particle flux, but it is not yet clear how the preamplificalion zone behaves at high rates. Although the construction of the chamber becomes more complex, this scheme may be applied to the extremely high rate at small angles.

\section{Gain Saturation}

For the $\mathrm{dE} / \mathrm{dx}$ measurement, we have to operate the chamber strictly in the proportional region. For a better spatial resolution, a bigger avalanche size is pre- 
ferable. Therefore, the maximum avalanche size in the proportional region should be chosen for actual experimental conditions. For ${ }^{55} \mathrm{Fe} x$-rays, the deviation from linearity is about $5 \%$ at $q_{o}=2 \sim 3 \times 10^{6} \mathrm{e}$. This gain saturation can be explained by the local distortion of electric field around the avalanche due to the local space cisarge. For typical chambers, the field on the surface of the anode is $2 \times 10^{5} \mathrm{v} / \mathrm{cm}$. On the other hand, the field on the surface of the sphere of positive ion space charges $q_{0}$ is $E_{r}=1.7 \times 10^{-7} q_{o} / r_{D}^{2}(V / c m)$, where $r_{D}$ is the radius of the positive ion cloud. $r_{D}$ is deternined by such factors as the distribution of primazy ionization diffusion of drifting electrons and avalanche spread near the anode wire. For ${ }^{55} \mathrm{Fe}$, x-rays, the main contribution is the track length of emitted electrons and $r_{D}$ is about $150 \mathrm{~mm}$. Then a field distortion of $1 \%$ is obtained at $q_{0}=2.7 \times 10^{6} \mathrm{e}$ which corresponds to a gain variation up to $5 \%$.

For charged particles crossing the chamber, this local space charge effect depends strongly on the position and angle of the particle path with respect to the anode wire. The worst case occurs when particles cross the chamber at the position of the anode wire with an angle of $90^{\circ}$ and leave an ionization chain which drifts toward the same spot on the anode wire. The avalanche due to the electrons which arrive first may produce a space charge and reduce the original multiplication field. For simplicity, we assume that the half of the primary electrons in a $5 \mathrm{~mm}$ gap contribute to build up the space charge. Other electrons arrive after $60 \mathrm{~ns}$. At this moment, positive ions move away about $90 \mathrm{~mm}$ from the. anode wirz. Therefore, the diameter of the positive ion cloud ${ }^{r} \mathrm{D}$ is $\sim 100 \mu \mathrm{m}$. $1 \%$ dis:azion of the field is obtained at $q_{0}=1.2 \times 10^{6} \mathrm{e}$, and the total avalanche size wo: be $q_{0}=4.8 \times 10^{6} \mathrm{e}$ because ionizations on both sides of the gap are collected the same anode. These values are in reasonable agreement with observations in rea: chambers. In most experiments proposed for ISA, the tracks are naturally inclined with respect to the anode wire and therefore, the proportional region would be extended. 
Assumed value of $q_{0}=2 \times 10^{6} \mathrm{e}$ in the previous sections seems to be reasonable as an optinum for both the accurate position readout and the accurate $\mathrm{dE} / \mathrm{d} x$ measurement.

\section{Radiation Hardness of Electronics}

Sirce same electronics for the chamber is also placed in the high radiation environment, radiation hardness of those IC's is an important factor for the life time of the detector system. Bipolar electronics can accept about $10^{6}$ rad, where the $\beta$-value shifts by $5 \%$. Commercial CMOS can accept $10^{4}$ rad and $10^{5}$ rad is possible by moderate hardening. Further improvement is possible (up to $2 \times 10^{6}$ rad) at much higher cost. 1 rad is estimated to be equivalent to $3 \times 10^{7}$ minimum ionizing particles per $\mathrm{cm}^{2}$ and the corresponding number for $10^{6}$ rad is $3 \times 10^{13}$ particles $\cdot \mathrm{cm}^{-2}$. At $r=10 \mathrm{~cm}$, the particle rate is about $3 \times 10^{4} \mathrm{~cm}^{-2} \cdot \mathrm{s}^{-1}$ and the expected 1 ife time is about 30 years. This value is comparable or even better than the chamber life because electronics can be placed relatively far from the bean axis.

Concluding, one can choose suitable configurations of chamber geometries, proportional and drift chambers can be used for accurate particle tracking at ISABELLE with $\mathrm{L}=5 \times 10^{32} \mathrm{~cm}^{-2} \mathrm{~s}^{-1}$. Chambers can be operated at reasonable avalanche size of $q=2 \times 10^{6} \mathrm{e}$, thus both the spatial resolution, as shown below, and the thergy resolution will be kept at an acceptable level.

$$
\text { H.B.J., H.O., A.H.W. }
$$

\section{RESOLUTION OF DRIFT CHAMBERS}

\section{Time Sesiution}

I: $=$ =ein contributions to the resolution of drift chambers are due to:

1. The time resolution of the electronic readout system.

2. Statistical fluctuations in the production of the primary ionization along the particle's path. 


\section{Electron diffusion during the drift time.}

Because of the high rates expected for ISABELLE, it is essential to operate the chambers at very low gas gain. The resolution of drift chambers is discussed under tiis aspect. The electronics time resolution is determined by the equivalent noise charge $\left(\overline{E N C}^{2}\right)^{\frac{1}{2}}$ and the slope of the leading edge of the signal ds/dt

$$
\delta t=\frac{\left(\overline{E N S}^{2}\right)^{\frac{1}{2}}}{d s / d t}
$$

The noise depends on detector parameters as input capacity $C_{i n}$, resistance $R_{p}$ paraliel to the input, on the type of amplifier and the characteristics of the pulse shaping circuit.

The total noise is mainly the quadratic sum of two terms, the series equivalent noise charge $\left(\overline{E N C}_{s}^{2}\right)^{\frac{3}{2}}$ and the parallel equivalent noise charge $\left(\overline{E N C}_{\mathrm{p}}^{2}\right)^{\frac{1}{2}}$ (see Ref. 10 for more details).

$$
\overline{\operatorname{ENC}_{S}^{2}}=\frac{2}{2} a_{F 1} \cdot \frac{e_{n}{ }^{2} C_{i n}{ }^{2}}{t_{r}}
$$

where

$$
\begin{aligned}
& e_{n}=\text { rms eqiv. series noise voltage per } \mathrm{Hz}^{\frac{2}{2}} \\
& a_{F 1}=\text { filter form factor } \\
& t_{I}=\text { signal rise time after filter } \\
& \qquad \frac{E N C_{p}^{2}}{E}=\frac{4}{3} \mathrm{kT} \frac{t_{\mathrm{m}}}{R_{p}}
\end{aligned}
$$

where

$\mathrm{K}_{\mathrm{p}}=$ parallel noise resistance

The cijpping time $t_{m}$ will be for the purpose of time measurement of the order of the $\sum_{\equiv \equiv}$ : rise time of the signal $t_{m}=t_{r}=25 \mathrm{~ns}$. For a fall time of the signal $t_{f}=5 t_{I}$ one obtains $a_{F 1}=1.2$.

Three cases will be compared with the amplifier connected to a single anode wire resulting in $C_{\text {in }}=25 \mathrm{pF}$ ( Table 3 ). 
- charge sensitive amplifier (FET-input)

- coinmon base amplifier (bipolar transistor)

- common base amplifiers on both ends of a resistive anode wire( $R_{a}=2.2 \mathrm{k} \Omega$ ) for position determination along the wire using charge division. ${ }^{11}$

The comon base input stage has the advantage to. provide a fast current signal which requires very little further shaping and it is simple in construction and operation. However, under certain conditions the low equivalent parallel noise resistance produces considerably more noise than a charge sensitive amplifier.

For the signal, conservative assumptions are made. After $25 \mathrm{~ns}$, the positive ions have traveled $50 \mu$ and the fraction of induced charge is 0.20 of the total charge $Q_{0}$. Since one is interested in measuring the arrival of the "first electron", only about $1 / 10$ of the total ionization in a $1 \mathrm{~cm}$ gap is useful. Therefore, the induced charge to be considered after $25 \mathrm{~ns}$ is $Q=0.02 Q_{0}$. Assuming an appropriate avalanche. size of $Q_{0}=2.10^{6}$ electron charges for the ISA environment and the rise time of the signal to be about two times faster than the linear approximation, one obtains ds/dt $=3.2 \times 10^{3}$ electrons/ns.

Table 3 shows that in all cases the time resolution will be well below 1 ns which corresponss $\left(v_{\text {drift }}=5 \mathrm{~cm} / \mathrm{us}_{\mathrm{s}}\right)$ to an equivalent position error of $\sigma<50 \mu \mathrm{m}$.

Table 3. Equivalent Noise Charges (Electrons)

\begin{tabular}{|c|c|c|c|c|c|}
\hline 8 & $\mathrm{R}_{\mathrm{p}}(\Omega)$ & $\left(\overline{E N C}_{p}^{2}\right)^{\frac{1}{2}}$ & $\left(\overline{\mathrm{ENC}}_{\mathrm{s}}^{2}\right)^{\frac{1}{2}}$ & $\left(\overline{E N C}_{\text {tot }}^{2}\right)^{\frac{1}{2}}$ & $\delta t(n s)$ \\
\hline Charge-3e-isive (FET) & $>10^{5}$ & $<2.10^{2}$ & $1.0 \cdot 10^{3}$ & $1.0 \cdot 10^{3}$ & 0.3 \\
\hline Common $t \equiv \equiv \equiv$ (bipolar) & $4.3 \cdot 10^{3}$ & $1.12 \cdot 10^{3}$ & $1.0 \cdot 10^{3}$ & $1.5 \cdot 10^{3}$ & 0.5 \\
\hline Common base - charge d & $1.46 \cdot 10^{3}$ & $1.9 \cdot 10^{3}$ & $1.0 \cdot 10^{3}$ & $2.2 \cdot 10^{3}$ & 0.7 \\
\hline
\end{tabular}


The time resolution is a function of the avalanche size and, therefore, strongly coupled to considerations of life time, saturation effects, rates, etc.

The statistical fluctuations and electron diffusion are clearly dependent on the gas used in the drift chamber and determine the intrinsic spatial resolution of the device. The statistical fluctuations are particularly important near the anode wire where the resolution is limited by the mean distance between primary clusters. For some drift chamber geometries, the resolution near the field wires is also reduced by this effect.

In general, farther from the anode wire the dominant effect will be due to the electron diffusion. For a drift distance of $\sim 5 \mathrm{~mm}$, this contribution to the spatial resolution is of the order of 50 to $70 \mu \mathrm{m}$ and is proportional to the square root of the drift distance. For the relatively short drift distances that will be required for the IS $A$ environment, the intrinsic spatial resolutions of drift chambers can be less than $\sim 100 \mathrm{~mm}$. This intrinsic resolution seems to be somewhat far larger than the electronic time resolution. However, the numbers quoted for the amplifiers are optimum values, which in practice may not be reached, in particular, because at the high frequency considered the circuits behave rather complex, so that one can expect overall spatial zesolutions of the order of 100-200 $\mathrm{mm}$. For a very large number of channels the circuits certainly will be simpler and the upper part of the range has to be considered.

\section{Double Puise Resolution}

The rxititrack resolution of drift chambers require the capability of recording the apg:atiate number of hits per wire in an event and depends on the electronic occupation :ime of the amplifier-discriminator as well as the intrinsic time and space reszitions of the chamber itself. Electronically, a double track resolution 
of better than $1.5 \mathrm{~mm}$ can be achieved, ${ }^{12}$ and systems ${ }^{13}$ presently exist for recording multiple hits on a single wire with comparable time resolutions.

However, in a drift chamber operated in the proportional mode one cannot distinguish a second avalanche unless it arrives after all the clusters from the first track have been collected. This collection time is typically 100-200ns and corresponds to a double track resolution of the order of 5-10 mm.

By using relatively thin chambers and perhaps operating at high gas gains, one can improve the double track resolution to some degree, especially for tracks incident nornally to the anode plane. Double track resolutions of the order of $2 \mathrm{~mm}$ (40 ns) have been achieved with this method. Another way toobtain good double track resolution may be simply to reduce the cell size by adding electronegative gas to a multiwire proportional cluster. Including an appropriate number of such proportional and/or drift chambers in the detector would improve the double track resistance of the whole system.

Momentum Resolution and Multiple Scattering

Consider a system of $n+1$ parallel drift chambers uniformly spaced in a uniform magnet field that is parallel to the anode wires. The momentum resolution due to the spatial resolution of the chambers is: ${ }^{14}$

$$
\left(\frac{\delta P_{0}}{P_{0}}\right)^{2}=f(N)\left(\frac{P \tau_{x}}{B I^{2}}\right)+g(N)\left(\frac{\tan \lambda}{1+\tan ^{2} \lambda} \cdot \frac{\sigma_{z}}{L}\right)^{2}
$$


where: $\mathrm{p}_{\mathrm{O}}=$ total momentum

$\mathrm{p}=$ component of the momentum normal to the magnetic field

$B=$ magnetic field strength

$\mathrm{L}=$ distance between the first and last chambers

$\lambda=$ compliment of the angle between $P_{0}$ and $B$

$-\sigma_{\overline{\mathbf{x}}}=$ spatial resolution_in the drift direction

$\sigma_{z}=$ spatial resolution along the anode wire

$f(N) \approx 720 / N+5$

for $\mathrm{N}^{2} \gg 1$

$g(N) \approx 12 / N+3$

For 16 chambers at $10 \mathrm{~cm}$ intervals $(\mathrm{L}=1.5 \mathrm{~m}$ ) in a 20 kilogauss magnetic field with $\sigma_{x}=.2 \mathrm{~mm}, \sigma_{z}=2 \mathrm{~cm}$ and $\lambda=45^{\circ}$,

$$
\left(\frac{\delta \mathrm{p}_{\mathrm{o}}}{\mathrm{p}_{\mathrm{o}}}\right)^{2}=\left(\frac{\mathrm{p}}{1100 \mathrm{GeV}}\right)^{2}+(.0054)^{2}
$$

One notes țhat for particles having momenta greater than $\sim 10 \mathrm{GeV}$, the momentum resolution is not seriously degraded by a much lower spatial resolution in the magnetic field direction even for tracks at quite large angles relative to the bending plane. This is particularly appropriate to "bicycle wheel" detectors where " one usually relies on two dimensional readout methods to measure the coordinate parallel to the anode wires (see below).

For tracks that are normal to the magnetic field the uncertainty in the momentum measurement due to multiple scattering is approximately:

$$
\left(\frac{\delta_{\mathrm{p}}}{\mathrm{p}}\right)_{\text {M.S. }}=h(\mathrm{~N}) \frac{0.015 \mathrm{GeV}}{\beta \text { B L }} \sqrt{\ell / l_{\mathrm{rad}}}
$$

where $l / l^{-r a d}=$ number total radiation lengths assumed to be equally distributed

$$
\begin{aligned}
& \hat{s}=v / c \approx 1 \\
& h(N) \quad 1.2 \text { for large } N .
\end{aligned}
$$


For $B=20$ kilogauss and $L=1.5 \mathrm{~m}$,

$$
\left(\frac{5 \mathrm{p}}{\mathrm{p}}\right)_{\text {M.S. }}=0.02 \sqrt{\ell / \ell_{\mathrm{rad}}}
$$

Table 4 stows the effect of the multiple scattering on the momentum resolution for the 16 chamber system considered above for three types of chamber construction. It was assimed that there was air between the chambers.

Also included are the effects of $1.5 \mathrm{~m}$ of the gasses that have been suggested as being appropriate for $\mathrm{dE} / \mathrm{dx}$ counters. The propane fillings are comparable to conventional chambers of wire and thin foil construction. The Xe and high pressure A are somewhat worse but still compatible with conventional foil chambers. However, ten atmospheres of $\mathrm{Xe}$ is approximately 1 radiation length and would have considerably. more multiple scattering than conventional chambers.

\section{Table 4}

\section{Momentum Resolution Due to Multiple Scattering}

\section{Material}

16 typical chambers (.001 r.1.)

16 foam chambers (.004 r.1.)

16 thin foil chambers (.0002 r.1.)

$1.5 \mathrm{~m} \mathrm{~A}: \mathrm{atm})$

$1.5 \mathrm{~m} A(2 \mathrm{~atm})$

$1.5 \mathrm{~m} \because:::$ atm)

$1.5 \mathrm{~m}: \because: 0$ atm)

$1.5 \mathrm{~m} P:=\vdots \approx \mathrm{se}$ ( $1 \mathrm{~atm}$ )

$1.5 \mathrm{~m} \equiv=\because \equiv 2 \mathrm{c}$ (3 atm) $\ell / l_{\mathrm{rad}}$

.020

.065

.009

.014

.135

.100

1.00

.0065

.020 $\left(\frac{\delta p}{p}\right)$ M.S.

.005

.002

.002

.007

.006

.020

.002

.003 


\section{Table 5}

Momentum Resolution Due to Chamber Resolution

Number of Samples in $1.5 \mathrm{~m}$

16

56

448 $\left(\frac{\delta \mathrm{p}}{\mathrm{p}}\right)_{\operatorname{Res}}\left(\mathrm{GeV}^{-1}\right)$

$\mathrm{p} / 1100$

$p / 1950$

$\mathrm{p} / 5350$

For comparison, Table 5 shows the momentum resolution for the 16 chamber system considered above and for the 56 and 448 sample systems that were considered for $\mathrm{dE} / \mathrm{dx}$ measurements. A chamber resolution of $.2 \mathrm{~mm}$ was assumed. It is clear, for example, that for a Xe $\mathrm{dE} / \mathrm{dx}$ chamber at 10 atmospheres, one does not need $.2 \mathrm{~mm}$ resolution for momentum determination since for such a chamber multiple scattering dominates the momentum resolution for momenta up to $\sim 40 \mathrm{GeV}$ $(\sim 100 \mathrm{GeV})$ for 56 (448) samples.

More generally, the effect of the multiple scattering on the momentum resolution can be seen by considering the conditions and the momentum $p *$ under which the multiple scattering and the spatial resolution contribute equally to the momentum determination. Under these conditions (for $N+1$ uniformly spaced samples),

$$
\begin{aligned}
& \mathrm{p}^{*} \approx 6.7 \times 10^{-4} \frac{\mathrm{L}}{\sigma_{\mathrm{x}}} \sqrt{\ell / l_{\mathrm{rad}}(\mathrm{N}+5)}(\mathrm{GeV} / \mathrm{c}) \\
& \text { for } I=1.5 \mathrm{~m} \text { and } \sigma_{x}=200 \mu \text {, one obtains } \\
& P^{*} \approx 5 \sqrt{l / l_{\operatorname{rad}}(N+5)}(\mathrm{GeV} / \mathrm{c})
\end{aligned}
$$


Summarizing, it is found that the high number of cells (typically 100) required for the $\mathrm{dE} / \mathrm{dx}$ measurement also have considerable influence on the momentum resolution. A position resolution of $\sigma \approx 200 \mu$, which is easily obtained $\because i t i$ an avalanche size of $Q_{0} \approx 2.10^{6}$ electrons, gives already excellent momentua resolution. However, the considerable thickness of the pressurized counting gas introduces such a strong multiple scattering that it may limit the momentum resolution below $5 \mathrm{GeV} / \mathrm{c}$ if high $\mathrm{z}$ noble gases are used..

$$
\text { R.S., A.H.W., C.D.W }
$$

TWO DIIENSIONAL READOUT

Two dimensional readout ideally reduces the chamber data to points in 3 dimensions with good accuracy in all coordinates. These are reconstructed from a constrained triplet of time and/or pulse height information in the anode wire plane. Here we summarize known methods of $2-D$ point localization according to spatial accuracy, electronics requirements, and multihit capability.

The latter means specifically to determine the probability of reconstruction of a multihit event. For simplicity we consider first only the reconstruction in space, the time distance between events being large compared to the memory time of the system. The high rate behavior can then be deducted easily by substituting the hit multiplicity of a single event by the apparent hit multiplicity within the memory tine, adding random hits.

\section{Delay iris}

Tr: s-trinsic resolution given by the noise analysis of Radeka ${ }^{10}$ is:

$$
\therefore 2 / 8=2.46 \cdot \frac{{ }^{T} F W}{T_{D}} \frac{e_{n}}{Z_{O} Q_{0} \varepsilon} T_{F W}^{\frac{1}{2}}
$$


where: $\quad T_{F W}=$ the time dispersion of the line

$$
\begin{aligned}
\tau_{D} & =\text { the total delay time } \\
e_{n} & =\text { the noise voltage } / \sqrt{\mathrm{HZ}} \\
Q_{S}=\varepsilon \cdot Q_{0} & =\begin{array}{l}
\text { the charge induced on the delay within } \\
\text { the collection time. }
\end{array}
\end{aligned}
$$

For $\tau_{D} / \tau_{F W}$, values ranging from 6 to 14 have been measured. $15,16,17,18$ We use 10 as a standard value. To obtain the expected resolution from this technique, we set $z_{0}=500 \Omega, e_{n}=1 \mathrm{nV} \cdot / \sqrt{\mathrm{HZ}}, \mathrm{T}_{\mathrm{FW}}=50 \mathrm{~ns}$ and assume $100 \%$ coupling efficiency. Then

$$
\frac{\delta l}{1}=\frac{1.1 \times 10^{-16}}{\varepsilon \cdot Q_{0}}
$$

and for an avalanche size of $2.10^{6}$ electrons with a collected charge in 200 ns of $6.10^{5}$ electrons, one obtains $\delta / / \ell=1.10^{-3}$. Simple helical delay lines have been reported ${ }^{17}$ giving results compatible with Eq. (18).

In practice, coupling efficiency is of the order of $30 \%$ but larger coupling could be achieved by, for example, surrounding the drift cell with the windings of the delay line. 18

One channel of delay Iine readout is required per drift cell. Printed circuit delay lines have been used $^{19}$ with a sense wire doublet to remove leftright ambiguities with doublet spacings as small as $6 \mathrm{~mm}$. However, up to now, the spatial resolution is lower than in helical delay lines at the same avalanche size.

The occupation time of an anode pulse, ${ }^{\top} A$, gives rise to a dead region in the drift cell. The width is given by $\tau_{A} \cdot V_{D}$, with $V_{D}$ the drift velocity. The length is clearly the length $l$ of the anode wire and one loses a strip of area ${ }^{T} V_{D} l$, which is parallel to the anode wire. The dead region corresponding to a 
delay line pulse of occupation time $\tau_{D L}$ is a strip of area $\tau_{D L} \cdot V_{D} \cdot \ell$ crossing the dead area of the anode at an angle of $\theta=\tan ^{-1}\left(V_{D} / V_{D L}\right)$. For a constrained triplet of time measurements, geometry gives the additional dead area.incurred by this method and a fast delay line is clearly preferred. We take $\tau_{A}=\tau_{D L}=$ $100 \mathrm{~ns}, \ell=1.5 \mathrm{~m}$ and $v_{D}=5 \mathrm{~cm} / \mu \mathrm{s}$. Then one loses an additional $40 \%$ at $v_{D L}$ of 1. $\mathrm{cm} / \mathrm{ns}$ which increases to $160 \%$ for $\mathrm{V}_{\mathrm{DI}}=.1 \mathrm{~cm} / \mathrm{ns}_{\mathrm{s}}$.

\section{Charge Division}

Here, the limiting spatial resolution is

$$
\frac{\delta \ell}{l}=2.54 \frac{\left(k T C_{D}\right)^{\frac{1}{2}}}{Q_{S}} \cdot\left(\frac{\tau_{F}}{R_{D} C_{D}}\right)^{\frac{1}{2}} \cdot \sqrt{2} .
$$

where $Q_{S}$ is the signal charge within $T_{F}$ and $C_{D}$ is the anode wire capacitance. For the anode resistance, $R_{D}$, and the shaping time, $\tau_{F}$, we take the values given in Ref. 11 of $2.2 \mathrm{k} \Omega$ and $.25 \mathrm{ks}$, respectively, which gives

$$
\frac{\delta l}{l}=\frac{2 \cdot 3 \cdot 10^{-16}}{Q_{s}}
$$

This calculation, which implies $1.6 \%$ at an avalanche size of $Q_{0}=2.10^{6}$ electrons $\left(Q_{S}=0.45 Q_{0}\right)$ agrees with the measurements.

In addition to its simplicity of construction, this method clearly lends itself to pulse height measurement. If this information is not required, unconventional electronics such as civicing analog-to-digital converters could be used.

Left-right ambiguities are not resolved unless one staggers successive wires. 20

There is, in principle, no loss in multihit capability with this method beyond the loss given by the occupation time of the anode pulse $\tau_{A}$. Tests with preamplifiers with common base input indicate that pulses can be shaped for high gas gain to 65 ns. 


\section{Cathode Strips}

Here, one segments the cathode planes and uses these strips to sample the induced pulses which are distributed with a full width at half maximum of approximately IWFM $=0.5 \mathrm{~d}$ with $\mathrm{d}$ the gap width. 21,22 To estimate the noise contribution to the resolution, we assume the charge to be measured over $k$ strips of width $d / 2$ (for simlicity we consider $k$ even), each.with a noise $Q_{\text {rmis }}$ and obtain.

$$
\delta \ell=\frac{1}{2} \frac{Q_{r m s}}{Q_{s}} d \sqrt{2 \sum_{n=1}^{n=k / 2} n^{2}} .
$$

Inducing half the avalanche charge in each cathode plane over four strips $(k=4)$ and measuring $34 \%$ of this within a collection time of $100 \mathrm{~ns}$, we find with $\mathrm{Q}_{\mathrm{rms}}=$ $4.5 \cdot 10_{\mathrm{e}_{0}}^{3}$ (charge-sensitive amplifier gives only about $10 \% 1$ less than the common base input stage) $\delta l \approx 0.3 \mathrm{~mm}$ for an avalanche size $Q_{0}=2.10^{6} \mathrm{e}_{0}$.

To evaluate the multihit capability of this scheme, we assume the, anode plane to be $1 \mathrm{~m}^{2}$, composed of $1 \mathrm{c}$.drift cells and the cathode strips have angles of \pm 45 with respect to the anode wires. In order to resolve ambiguities, one has three possible alternatives for correlating information:

- time correlation ${ }^{21}$ of cathode planes

- amplitude correlation ${ }^{23}$ of cathode planes

- measurement of additional coordinate from anode readout ${ }^{24}$.

The Eime correlation is limited by the signal to noise ratio which can be calculazed in the same way as for the anode signal (Eqs. (9), (10), (11)), only the higher capacity $\left(C_{\text {in }} \approx 200 \mathrm{pF}\right)$ gives a higher noise. With $Q_{\mathrm{rms}}=8 \cdot 10^{3} \mathrm{e}$, and only hal the charge as on the anode $\mathrm{ds} / \mathrm{dt}=1.6 \cdot 10^{3} \mathrm{e} / \mathrm{ns}$, the time resolution becores $\dot{z}=5 \mathrm{~ns}$. A correlation would be allowed for $\pm 3 \delta$ which defines a $t$ ime intervis of $30 \mathrm{~ns}$. If the maximum drift time is $100 \mathrm{~ns}$, in about $1 / 3$ of all cases, 
no correlation can be found. Subdividing the cathode strips in shorter pieces would considerably reduce this number $(25 \mathrm{~cm}$ long strips would give only $8 \%$ confusion).

For the method of amplitude correlation, physical processes are responsible for its limitation. Naively, one would expect that the two charges induced from the same positive charge at the anode wire are the same on both cathode planes. However, the fluctuation of ionization above and below the anode plane gives different induced charges on the upper and lower cathode because of the top/bottom asymmetry of the induced charges. ${ }^{22}$ Measuring an induced pulse on one cathode one has to allow a variation of $10-20 \%$ for the corresponding pulse on the other cathode, depending on gas gain and geometry. Since the total possible variation is of the order of $50-80 \%$ (Landau distribution), this method does not resolve the ambiguity in $25 \%$ of the cases.

If one uses the anode wire as a third coordinate, the resolving power of ambiguities is given by the total dead area for a single hit which is

$$
A_{d}=l\left(4 d+T_{A} V_{D}\right)
$$

since the induced pilse is spread over 4 strips, each $d / 2$ wide. The ratio of the dead area to the total area then gives the probability for unresolved ambiguities. For $\tau_{A} \cdot v_{D} \approx d$, one obtains for this ratio $1: 20$ which gets worse for smaller chambers. This nuber is also the practical limit for the timing method for obvious reasons.

\section{Conclusions}

For a ehamber of $1 \mathrm{~m}$ length, a resolution of 1 mon or less can be obtained with a delay line or cathode strips at an avalanche size of $2.10^{6}$ electrons. ... arrent division under such conditions gives a resolution of about 2 cn. Ultimately, the best multihit capability would be obtained using the current division method. 
For the delay line, this is slightly worse ( $40 \%$ additional losses). and for the cathode strip readout this depends strongly on the geometry. For the typical layout chosen, the losses are about a factor of 5 above the value for the current division. A. H.W., S.W.

Particle Identification Using $\mathrm{dE} / \mathrm{dx}$ Messurement

The investigation of the hadronic decay of the hypothetic $W$ or, more generally, the investigation of the quark-structure of hadrons, leads to the necessity of recording and analyzing jet-like events in detail. A particle analyzer of high modularity is needed. From the two candidates discussed at this moment, the "imaging Cerenkov counter" and the $\mathrm{dE} / \mathrm{dx}$ measurement in a multilayer track detector, the latter has the advantage that at least one working system exists 25 which gives confidence that the systems proposed for ISABELLE (clearly more ambitious) also would be feasible.

The system described in $\operatorname{Ref} .25$ samples the energy loss over $5 \mathrm{~m}$ of'track length, a value considered much too long for a central detector at ISABELLE since it would require a detector of $10 \mathrm{~m}$ diameter which has to be surrounded by the magnet coil, shower counters and $\mu$-identifier. Also, a wide angle spectrometer ${ }^{26}$ would not have enough space for a detector with this dimension. Therefore, in the 1977 ISABELLE Workshop, the question was raised ${ }^{27}$ whether sufficient particle resolution can be obtained for an effective track length of $1.3 \mathrm{~m}$ by optinizing all possible parameters: pressure, gas mixture, and number of subdivisions. The use of Ar at high pressure $(5 \ldots 10 \mathrm{~atm})$ or heavy hydrocarbons at somewhat lower pressure $(2 \ldots 3 \mathrm{~atm})$ seemed to be promising. In the meantime, a more detailed study has been undertaken ${ }^{28}$ which shows that substantial improvement under best conditions can be obtained, however, at the price of a finer subdivision (greater number of layers), higher total costs (if $\mathrm{Xe}$ is used) and reduced dynamic range of the momenta. 
Table 1

$\underline{k} / \pi$-separation at $p=3.5 \mathrm{GeV} / \mathrm{c}$

$\underline{\text { Gas }}$

$\mathrm{C}_{3} \mathrm{H}_{8}$

$\bar{I}=50.3 \mathrm{eV}$

$\rho=1.88 \mathrm{mg} / \mathrm{cm}^{3}$

$0.5 \mathrm{Ar}+0.5 \mathrm{C}_{2} \mathrm{H}_{6}$

$\bar{I}=100.8 \mathrm{eV}$

$\rho=1.46 \mathrm{mg} / \mathrm{cm}^{3}$

$0.9 \mathrm{Ar}+0.1 \mathrm{CH}_{4}$

$\bar{I}=191.2 \mathrm{eV}$

$\rho=1.56 \mathrm{mg} / \mathrm{cm}^{3}$

$0.875 \mathrm{Xe}+$

$0.05 \mathrm{C}_{3} \mathrm{H}_{8}+$

$0.075 \mathrm{CH}_{4}$

$\bar{I}=501.2 \mathrm{eV}$

$\rho=4.94 \mathrm{mg} / \mathrm{cm}^{3}$
Pressure

(atm) $\underline{\mathrm{n}} \underline{\mathrm{k} / \pi(\sigma)}$

1. $56 \quad 3.5$

$3 \quad 56,4.6$

$1448 \quad 4.6$

3448

6.8

156

10

56

$\left.\begin{array}{l}2.9 \\ 4.4 \\ 3.4 \\ 6.3\end{array}\right\}$

$1 \quad 56$

2.7

30

26 (7 atm)

30

26 (7 atm)

65

30 (5.5atm)

65

$30(5.5 \mathrm{~atm})$

10

448

6.8

3.4

$40(5.5 \mathrm{~atm})$

70

$66(5.5 \mathrm{~atm})$ 


\section{Conventional dE/dx}

Considering the time schedule of the ISABELIE project one may be allowed to call a method "conventional" which is just in the stage of its first successful applications. This method is the measurement of the relativistic rise of energy loss by means of subdividing the total absorber into a number of smaller cells, acting as-proportional counters, to measure the total charge produced in each cell and use the mean of the $40 \%$ smallest pulses as a final result.

The resolution of such a system can be characterized by the separation of these mean values for two particles $\left(E_{\pi}-E_{k}\right)$ (here $k / \pi$ separation is chosen) in units of the rms-fluctuation $\sigma$ of the mean value obtained for one particle.

Table 1 shows these numbers for a momentum of $p=3.5 \mathrm{GeV} / \mathrm{c}$ for a total track length of $\mathrm{L}=128.8 \mathrm{~cm}$, a subdivision into $\mathrm{n}$ layers and different typical gas mixtures. Where available, the high momentum behavior is characterized by two numbers. $\left(P_{\max }\right)_{R}$ is the momentum where the quoted resolution has dropped to 0.707 , of the value for $p=3.5 \mathrm{GeV} / c$. It gives the range relative to the best value. The other number $\left(\mathrm{P}_{\max }\right)_{A}$ gives the momentum where the $K$ and $\pi$ distribution intersect at half height $(\sigma=2.3)$ and gives the absolute range.

For each gas $\bar{I}$ the mean ionization potential and the density of a pressure of $1 \mathrm{~atm}$ and $20^{\circ} \mathrm{C}$ is shown. For higher pressure, the density increases linearly. The table reflects the general behavior ${ }^{28}$ that gases can be classified with respect to their mean ionization potential $\bar{I}$ and the density $\rho$. Better resolutions are obtained for high $\rho$, and either high or low $\bar{I}$. Pressure and subdivision dependence is such that finer subdivision has almost no influence at atmospheric pressure, but becomes very important at higher pressures. 
Unfortunately, the "best cases" have their particular disadvantage like restricted momentum range for dense hydrocarbons and high number of subdivisions, high cost, and a considerable total thickness (about 1 radiation length for pressurized Xe).

The systematics in the behavior of the gases investigated makes it unlikely that a "magic gas" may be found solving all problems at once.

In addition, the choice for gases working properly in a proportional wire chamber (this is even more true in a drift chamber) is restricted to a few gases and mixtures thereof.

The choice of the mixture and cell size depends, therefore, strongly on the experiment. If an excellent particle separation is vital, one will consider large pressure Xe in small subdivisions, hydrocarbons or provide more space for the detector. In the first generation experiment at ISABELLE, it may be sufficient to use an intermediate gas ( $\mathrm{Ar}-\mathrm{C}_{3} \mathrm{H}_{8}$, $\mathrm{Ar}-\mathrm{C}_{2} \mathrm{H}_{6}, \mathrm{Ar}-\mathrm{CO}_{2}$ or the like) in a detector with fine subdivisions (e.g., $4 \mathrm{~mm}$ ) but connect 3 or 4 wires together to reduce the number of readout channels. With this apparatus, a reasonable $\pi / k$ separation (about $4.5 \subset$ at $4 \mathrm{~atm}$ ) and a $\mathrm{k} / \mathrm{p}$ determination on a statistical basis $(2.6 \sigma)$ can be obtained. If it turrs out to be absolutely necessary, an improvement is possible by changing the gas, the pressure and the number of readout channels.

Unconvertional $\mathrm{dE} / \mathrm{dx}$

Tabi: 1 shows that for the "conventional" way of measuring $\mathrm{dE} / \mathrm{dx}$ the improvement in particle separation only can be obtained by increasing the pressure and therefoz: zestricting the dynamic range of momentum.

Fo: $\equiv$ sumber of physics programs at ISABELLE, however, it may be of impor tance to sep $\equiv \sum \equiv z$ a particles at higher momenta $(p>30 \mathrm{GeV} / \mathrm{c})$. A study at Fermilab 29 
showed that a combined transition radiation detector - $d E / d x$ system may be useful to solve this probler. It has been found that at very low pressure (Xe at about $30 \mathrm{~mm} \mathrm{Hg}$ ) the energy loss in a single cell (4 mm) had much smaller fluctuation, almost no tail as typical for the Landau distribution, and better particle separation than expected from extrapolation of the calculations at higher pressure $^{20}$ (see also Table 1). This study showed that, indeed, in the domain of aingle clusters the resolution may improve considerably and it may show the direction of further improvement.

Another approach in this direction is being investigated presently at BNL. 30 It is well known and has been frequently observed that a sharply differentiated anode signal shows several subsequent peaks for tracks crossing the chamber in the neighborhood of an anode wire. This structure is attributed to the statistical fluctuations of the primary ionization. Very large peaks may correspond to single $\delta$-rays (large clusters).

Two possible methods of obtaining a signal for particle identification based on the relativistic rise of the primary ionization are discussed here and compared to the standard method of measuring the total ionization (integrated pulse).

In principle, the number of clusters can be counted (see also Ref. 31) which would yield a poisson distribution with a much smaller variance than the distribution of total charge (Landau-distribution). Counting all clusters (in Ar at 1 atm one obtairs 20 clusters $(\mathrm{cm})$ would give an improvement over the integrating method by a facso: of about 2! Unfortunately, this is not possible with the present technois:y. One has to count at least 5 clusters/cm for the aforementioned gas in order to: :eak even with the integrating method, and an improvement is particularly difficu: electrons: as shown by a Monte Carlo calculation. 32 Until now, no serious attempt 
has been made to understand and improve the fast signal extraction in proportional counters but it may be rewarding to do so.

Another way of using the time structure of the differentiated signal consists of sampling the pulse height in small time bins (using a multi-threshold/ multi-shift register), discarding the big signals and using the integrated in- $\therefore$ formation of the (e.g., 40\%) smallest signals. This method represents the state of the art (at least in the ISABELLE time schedule) and gives an improvement over the standard method in the sense, that it permits the use of very fine sampling, while the number of chamber planes, wires and amplifiers remains reasonable. An "effective" gap width of about $2 \mathrm{~mm}$ (corresponding to $40 \mathrm{~ns}$ wide. time slices) seems reasonable and the data, as in Table 1 , can be used to estimate the improvement over the measurement in a $1 \mathrm{~cm}$ gap. Depending on gas and pressure, an irop rovement of a factor of 1.2 to 1.7 may be obtained.

\section{Conclusion}

The standard technigue of measuring the relativistic rise of ionization in gases (total ionization measurement) will give satisfactory results only at higher pressure. The better results can be obtained for lower mornenta ( $\mathrm{p} \leqq 15 \mathrm{GeV} / \mathrm{c}$ ) or. expensive $\mathrm{Xe}$ and a very large number of readout channels (of the order of $10^{4}$ ). Deeper insight into the ionization process and the signal formation may lead to a substantial improvezent, but further investigations are needed.

$$
\text { H.O. Y.T.; A.H.W. }
$$




\section{REFERENCES}

1. A. H. Walenta, "Proportional Chambers and Multiwire Drift Chambers at High Rates", Proc. of 1977 ISABELLE Summer Workshop, BNL 50721.

2. F. E. Paige, "Charged Particle Production at ISABELLE", Proc. of 1977 ISABELLE Summer Workshop, BNL 50721.

3. L. Rosenson, "Rate Limitations on Drift Wire Operating in the Proportional Mode", Proc. of 1977 ISABELLE Summer Workshop, p. 87, BNL 50721.

4. A. Etkin, "Rate Estimates at ISABELIE", Proc. of the 1977 ISABELLE Summer Workshop, BNL 50721 .

5. J. Fischer, S. E. Sobottka, and A. H. Walenta, to be published.

6. H. B. Jensen, private communication.

7. J. Fischer, H. Okuno, V. Radeka, and A. H. Walenta, to be published.

8. D. Nygren, private communication.

9. G. Charpak, G. Melchart, G. Petersen, F. Sauli, E. Bourdin, B. Blumenfeld, C. Duchazeaubeneix; A. Garin; S. Majewski, and R. Walczak, "New Approaches to High Rate Particle Detectors", CERN 78-05 (1978).

10. V. Radeka, IEEE Trans. Nucl. Sci., NS-21 (1974) 51.

11. V. Radeka and P. Rehak, IEEE Trans. Nucl. Sci., NS-25 (1978) 46.

12. A. Breskin, G. Charpak, F. Sauli, M. Atkinson, and G. Schultz, Nucl. Instr. and Meth. 124 (1975) 189. 
13. For a summary see: "Notes on Drift Chamber Time Digitizer Meeting", M. Atac and T. Droege, Fermilab Report TM-553.

14. Se $=$, e.g., R. L. Gluckstern, Nucl. Instr. and Meth. 24 (1963) 381.

15. R. Grove, I. Ko, B. Leskovar, and V. Perez-Mendez; Nucl. Instr. and Meth. $\underline{99}(1972) 381$.

16. CERN-Columbia-Oxford-Rockefeller Collaboration: "A System of Cylindrical Drift Chambers in a Superconducting Solenoid", Wire Chamber Conference, Vienna, Austria 1978.

17. H. Okuno, R. L. Chase, J. Fischer, and A. H. Walenta, IEEE Trans. Nucl. Sci., NS-24. (1977) 213 .

18. 0. Achtenberg, A. F. Garfinkel, G. Flügge, H. Jensing, W. Zimmermann, H. Meyer, M. Rössler, Wire Chamber Conference, Vienna, Austria 1978.

19. M. Atac, R. Bosshard, S. Erhän, and P. Schlein, IEEE Trans. Nucl. Sci. NS-24 (1977) 195.

20. J. Heintze, "Drift Chambers and Recent Developments", Wire Chamber Conference, Vienna, Austria 1978; C.'W. Fabjan, J. Lindsay, F. Piuz, F. Rangard, E. Rosso, A. Kulge, S. Serednyakow, W. J. Willis, H. P. Jensen, and J. D. Petersen, conerbution to the Wire Chamber Conference, Vienna, Austria 1978.

21. A. Valenta, "Lokalisierung von Teilchenspuren dunch Messung der Ei

22. J. Es: sner, H. Okuno, and A. H. Walenta, Nucl. Instr. and Meth. 151 (1978) 451; A. :. Kalenta, Nucl. Instr. and Meth. 151 (1978) 461. 
23. "CELLO - Proposal for a $4 \pi$ Magnetic Detector for Petra", DESY-Karlsruherïinchen-Orsay-Paris-Saclay, Fall 1976.

24. G. Fischer and J. Plch, Nucl. Instr. \& Meth. 100 (1972) 515.

25. I. Lehraus, R. Matthewson, W. Tejessy, and M. Aderholz, Nucl. Instr. \& Meth. $153(1978) 347$.

26. D. H. White, "Large Hadron Spectrometer Workshop Sumary", Proc. of 1976 ISABELLE Summer Workshop, BNL 50611.

27. J. Fischer, H. Okuno, and A. H. Walenta, "Measurement of Energy Loss in the Region of Relativistic Rise for Particle Identification", Proc. of 1977 ISABELLE Summer Workshop, BNL 50721.

28. J. Fischer, H. Okuno, A. H. Walenta, and C. L. Wang, to be published.

29. A. G. Oganessian, A. T. Sarkissian, and M. Atac, Nucl. Instr. \& Meth. 145 (1977) 251.

30. E. D. Platner, private communication.

31. J. H. Cobb, W. W. M. Allison and J. N. Bunch, Nucl. Instr. \& Meth. 133 (1976) 315.

32. V. C. Ermilova, I. P. Kotenko, and G. I. Merzon, Nucl. Instr. \& Meth. 145 $(1577) 555$ 


\section{FIGURE CAPTIONS}

Fig. 1. Chamber arrangement around an intersection region.

Fig. 2. Energy resolution of a proportional counter for ${ }^{55} \mathrm{Fe}$ over total accumulated charge/wire length.

Fig. 3. Configurations of grid wires. 


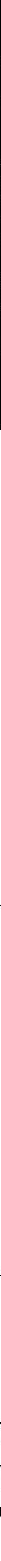




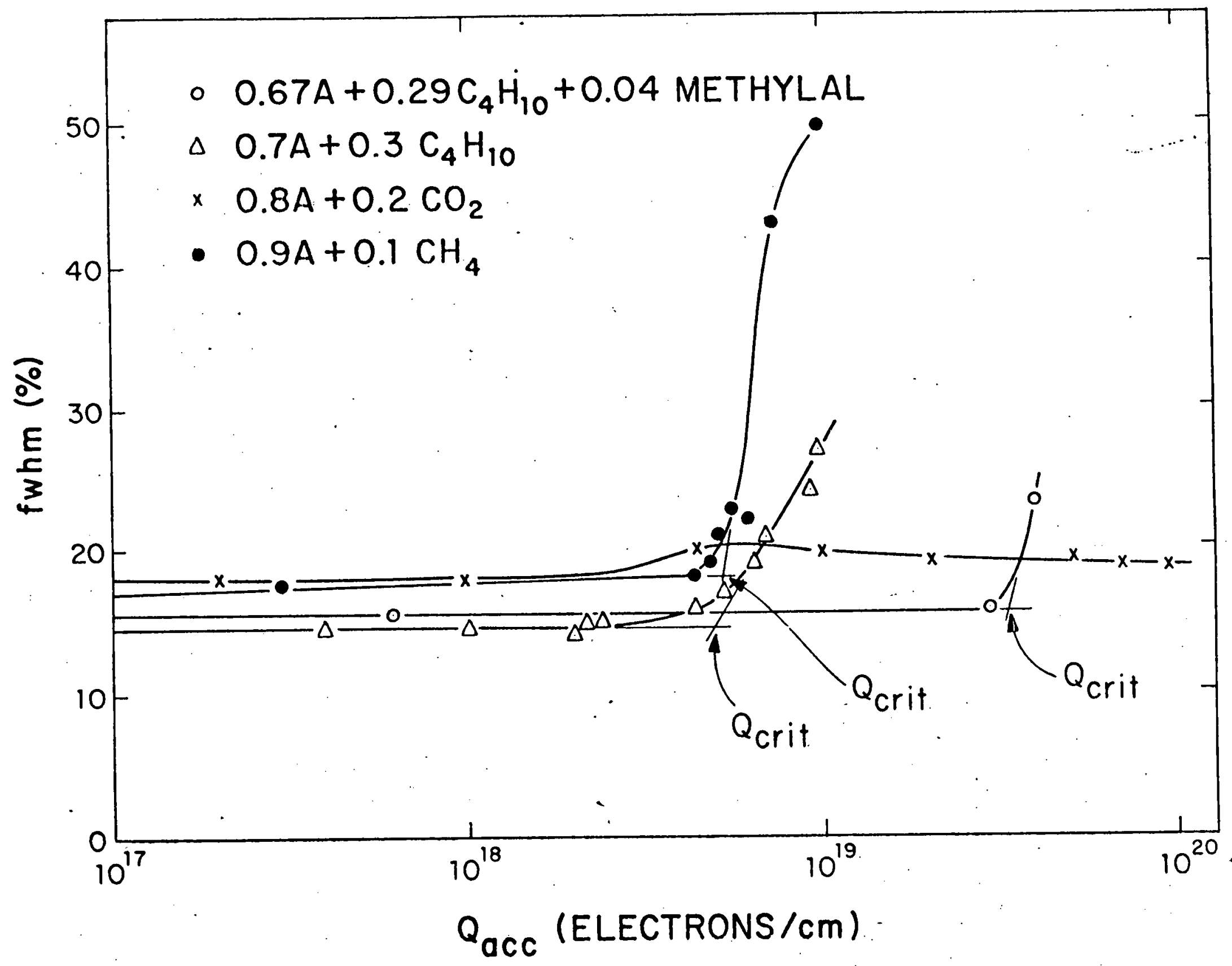




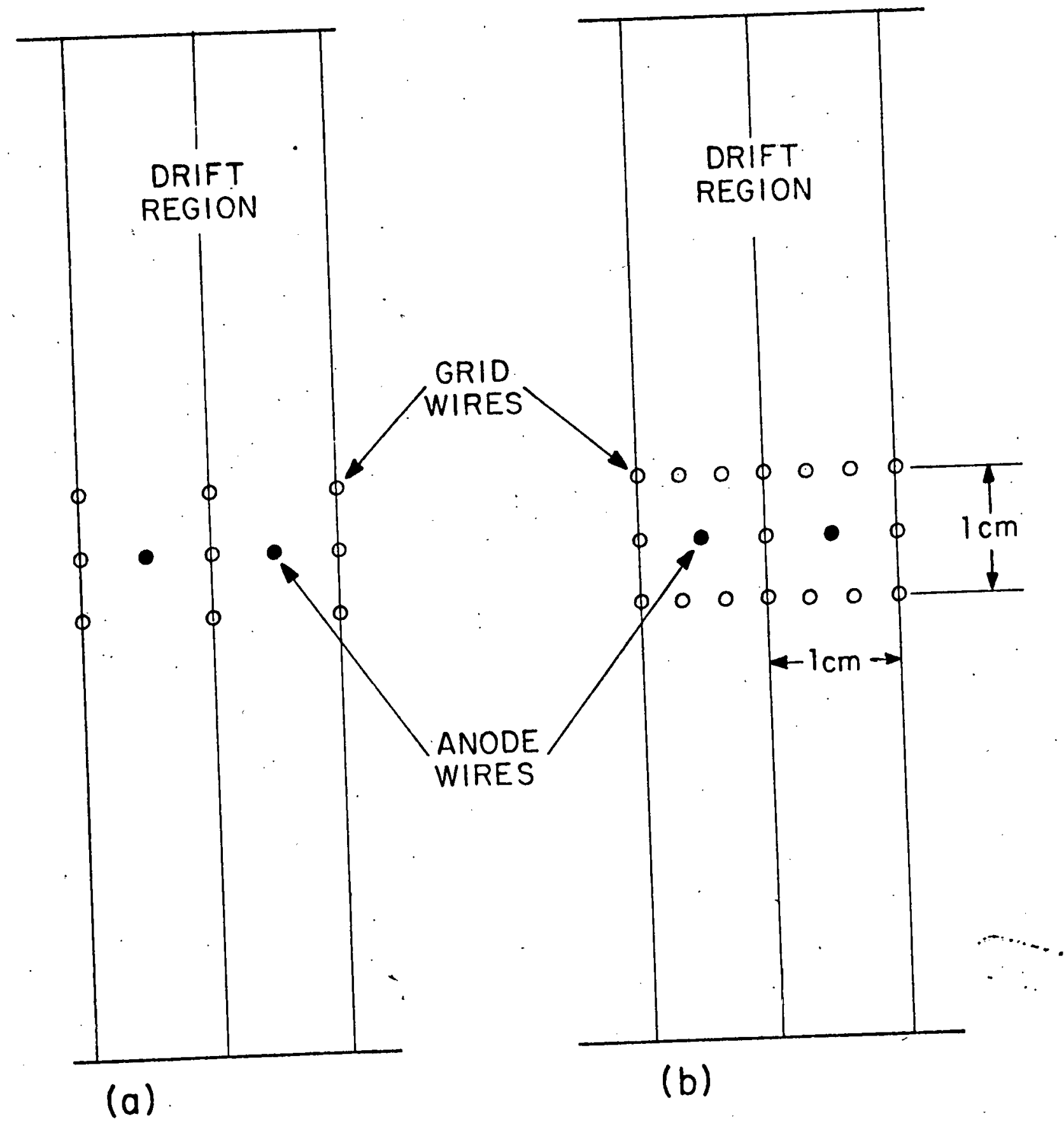

$\ldots$

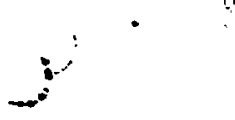

